\title{
A relation on 132-avoiding permutation patterns
}

\author{
Natalie Aisbett \\ School of Mathematics and Statistics, University of Sydney, Australia
}

received $9^{\text {th }}$ June 2013, revised $14^{\text {th }}$ Jan. 2014, $16^{\text {th }} \mathrm{Feb} .2014,24^{\text {th }}$ Mar. 2015, 15 $5^{\text {th }}$ May 2015, accepted $12^{\text {th }}$ Nov. 2015.

A permutation $\sigma$ contains the permutation $\tau$ if there is a subsequence of $\sigma$ order isomorphic to $\tau$. A permutation $\sigma$ is $\tau$-avoiding if it does not contain the permutation $\tau$. For any $n$, the popularity of a permutation $\tau$, denoted $A_{n}(\tau)$, is the number of copies of $\tau$ contained in the set of all 132-avoiding permutations of length $n$. Rudolph conjectures that for permutations $\tau$ and $\mu$ of the same length, for all $n$ we have $A_{n}(\tau) \leq A_{n}(\mu)$ if and only if the spine structure of $\tau$ is less than or equal to the spine structure of $\mu$ in refinement order. We prove one direction of Rudolph's conjecture by showing that if the spine structure of $\tau$ is less than or equal to the spine structure of $\mu$, then for all $n, A_{n}(\tau) \leq A_{n}(\mu)$. Furthermore, we show that $A_{n}(\tau)<A_{n}(\mu)$ when $n$ is greater than the length of $\tau$ and $\mu$. We disprove the opposite direction by giving a counterexample, and hence disprove the conjecture.

Keywords: permutations, permutation pattern, popularity

\section{Introduction}

A permutation $\sigma$ contains the permutation $\tau$ if there is a subsequence of $\sigma$ order isomorphic to $\tau$. In this case we say that $\tau$ is a pattern of $\sigma$. A permutation $\sigma$ is $\tau$-avoiding if it does not contain the permutation $\tau$. The popularity of a permutation $\tau$ in length $n 132$-avoiding permutations (defined more rigorously in Section 2), denoted $A_{n}(\tau)$, is the number of copies of $\tau$ contained in the set of all 132-avoiding permutations of length $n$. The question of pattern popularity within permutations that avoid some pattern was first asked by Joshua Cooper on his webpage [4]. Here he raised the question: what is the expected number of copies of a pattern in a randomly chosen pattern avoiding permutation? This problem was first tackled by Bóna in [1], where he proved that for all permutation patterns $\tau$ of length $k$ and for any $n \geq k$ we have

$$
A_{n}(12 \ldots k) \leq A_{n}(\tau) \leq A_{n}(k(k-1) \ldots 1) .
$$

He then extended this result in [2] to show that for all $n \geq 3$

$$
A_{n}(213)=A_{n}(231)=A_{n}(312) .
$$

In [5], Homberger studied Cooper's question for patterns of length 3 in 123-avoiding permutations.

Every 132-avoiding permutation $\tau$ has an associated binary tree $T(\tau)$ (defined in Section 4 which in turn has an associated partition called its spine structure. In [6], Rudolph extended the results of Bóna to show that:

1365-8050 @ 2015 Discrete Mathematics and Theoretical Computer Science (DMTCS), Nancy, France 
Theorem 1.1 ([6, Theorem 16]). For any 132-avoiding permutations $\tau$ and $\mu$ of length $k$, if $T(\tau)$ and $T(\mu)$ have the same spine structure, then for all $n \geq k$ we have $A_{n}(\tau)=A_{n}(\mu)$.

Chua and Sankar have since shown in [3] that if $A_{n}(\tau)=A_{n}(\mu)$ for all $n$, then $T(\tau)$ and $T(\mu)$ have the same spine structure.

In [6], Rudolph conjectured the following:

Conjecture 1.2 ([6, Conjecture 21]). Given patterns $\tau$ and $\mu$, for all $n$ we have $A_{n}(\tau) \leq A_{n}(\mu)$ if and only if the spine structure of $T(\tau)$ is less than or equal to the spine structure of $T(\mu)$ in refinement order.

In Theorem 4.6 we prove one direction of Conjecture 1.2. by showing that if the spine structure of $T(\tau)$ is less than or equal to the spine structure of $T(\mu)$ in refinement order, then $A_{n}(\tau) \leq A_{n}(\mu)$. It should be mentioned that Theorem 4.6 has previously been proven by Rudolph (unpublished). In Proposition 4.8 . we strengthen this result by showing that if $\tau$ and $\mu$ have length less than $n$ and the spine structure of $T(\tau)$ is less than the spine structure of $T(\mu)$ in refinement order, then $A_{n}(\tau)<A_{n}(\mu)$. Then, in Corollary 5.2. we disprove Conjecture 1.2 by showing that there exist permutations $\tau$ and $\mu$ such that for all $n$ we have $A_{n}(\tau) \leq A_{n}(\mu)$, whereas $T(\tau)$ and $T(\mu)$ are incomparable in refinement order.

We now give an outline of how the paper leads into the proof of the main theorem, Theorem 4.6. Chapter 2 introduces the main definitions that are used in the paper. Chapter 3 introduces a "move" that can be performed on a 132-avoiding permutation $\tau$, which gives another 132-avoiding permutation $\mu$ of the same length as $\tau$. Theorem 3.5 is used to show that $A_{n}(\mu) \geq A_{n}(\tau)$, and is the main theorem in Chapter 3 In Chapter 4 , we describe a well-known bijection between binary trees and 132-avoiding permutations. In this paper, this bijection sends the permutation $\tau$ to the tree $T(\tau)$. We describe this bijection in order to define the spine structure of 132-avoiding permutations, which is a property of their corresponding binary trees. Note that two distinct 132 -avoiding permutations may have the same spine structure. Then, in Proposition 4.4 we show that if $\phi$ is obtained from $\sigma$ by the popularity increasing move, then the spine structure of $\phi$ is greater than the spine structure of $\sigma$ in refinement order. We then show (see Proposition 4.5 and the proof of Theorem 4.6 that if the spine structure of a 132-avoiding permutation $\phi$ is greater than the spine structure of another 132-avoiding permutation $\sigma$, then there is a permutation $\tau$ with the same spine structure as $\sigma$, and a permutation $\mu$ with the same spine structure as $\phi$, such that $\mu$ is obtained from $\tau$ by the popularity increasing move. Since popularity is equal for permutations with the same spine structure (Theorem 1.1), this proves the main theorem of this paper, Theorem 4.6 .

\section{Acknowledgements}

I would like to thank my $\mathrm{PhD}$ supervisor Anthony Henderson for helpful conversations.

\section{Definitions}

Let $[n]$ denote the set of integers $\{1,2, \ldots, n\}$. Let $\sigma=\sigma_{1} \ldots \sigma_{n}$ denote a permutation of $[n]$ written in one-line notation. The permutation $\sigma$ contains the pattern $\tau=\tau_{1} \ldots \tau_{k}$ if there exist $1 \leq i_{1}<i_{2}<$ $\cdots<i_{k} \leq n$ such that for any $1 \leq s, t \leq k$ we have $\sigma_{i_{s}}<\sigma_{i_{t}}$ when $\tau_{s}<\tau_{t}$. A permutation pattern $\tau=\tau_{1} \ldots \tau_{k}$ is of length $k$. A 132-avoiding permutation is a permutation that does not contain the pattern 132. We denote the set of all permutations of $[n]$ that are 132 -avoiding by $S_{n}(132)$. 
Suppose that $\tau=\tau_{1} \ldots \tau_{k}$ is a permutation of length $k$ for some integer $k \leq n$, and suppose that $\sigma \in$ $S_{n}(132)$. Let $f(\sigma, \tau)$ denote the number of copies of $\tau$ contained in $\sigma$. For example, $f(53421,321)=7$ since the subsequences $532,531,542,541,521,321$ and 421 give rise to the pattern 321 . The popularity of a pattern $\tau \in S_{k}(132)$ in length $n 132$-avoiding permutations, denoted $A_{n}(\tau)$, is given by

$$
A_{n}(\tau):=\sum_{\sigma \in S_{n}(132)} f(\sigma, \tau) .
$$

Permutations $\tau$ and $\mu$ each of length $k$ are equipopular if for all $n, A_{n}(\tau)=A_{n}(\mu)$. Note that if a 132 -avoiding permutation contains a pattern, then that pattern must also be a 132 -avoiding permutation. There is $0 !=1$ permutation of the empty set, which is of length 0 . It certainly avoids 132 , and hence belongs to $S_{0}(132)$, so that $\left|S_{0}(132)\right|=1$. When $\tau$ is the length 0 permutation we take by convention $A_{n}(\tau)=\left|S_{n}(132)\right|$.

Suppose $\sigma_{1} \ldots \sigma_{n} \in S_{n}(132)$ and that $\sigma_{s_{1}} \ldots \sigma_{s_{k}}$ (where $s_{1}<\cdots<s_{k}$ ) gives rise to a permutation pattern $\tau$. Then we say that $\left\{\sigma_{s_{1}}, \ldots, \sigma_{s_{k}}\right\}$ are the elements in this occurrence of $\tau$, and for all $i \in[k]$ we say that $\sigma_{s_{i}}$ occurs as $\tau_{i}$.

\section{A popularity increasing move}

In this section we describe a move on 132 -avoiding permutations. We show that for all $n$, this move can be applied to any permutation in $S_{n}(132)$ other than $n(n-1) \ldots 1$, and that it results in another 132-avoiding permutation. We then show, in Theorem 3.5 , that this move produces a new permutation whose popularity is greater than or equal to that of the original permutation.

Observe that if $\sigma=\sigma_{1} \ldots \sigma_{n}$ is a 132-avoiding permutation, and if $\sigma_{j}=n$, then for all $i<j$ and for all $k>j$ we have $\sigma_{i}>\sigma_{k}$. In other words, all entries to the left of $n$ are greater than all entries to the right of $n$.

Suppose that $\sigma=\sigma_{1} \ldots \sigma_{n} \in S_{n}(132)$, and that there are a pair of indices $i, j$ with $i<j$, such that:

- $\sigma_{i}<\sigma_{j}$,

- for all integers $\alpha$ such that $\alpha<i$ we have $\sigma_{\alpha}>\sigma_{j}$,

- for all integers $\beta$ such that $i<\beta<j$ we have $\sigma_{\beta}<\sigma_{i}$.

Then we may form a new permutation $\phi=\phi_{1} \ldots \phi_{n}$, where $\phi_{1} \ldots \phi_{i-1}=\sigma_{1} \ldots \sigma_{i-1}, \phi_{i}=\sigma_{j}$, $\phi_{i+1} \ldots \phi_{j}=\sigma_{i} \ldots \sigma_{j-1}$, and $\phi_{j+1} \ldots \phi_{n}=\sigma_{j+1} \ldots \sigma_{n}$. In other words, $\phi$ is obtained from $\sigma$ by removing $\sigma_{j}$ and inserting it before $\sigma_{i}$. Since $\sigma$ is 132 -avoiding, all elements in $\left\{\sigma_{j+1}, \ldots, \sigma_{n}\right\}$ are either less than $\sigma_{i}$ or greater than $\sigma_{j}$, hence we must have $\sigma_{j}=\sigma_{i}+1$. We can therefore denote $\phi$ unambiguously by $\sigma^{\sigma_{j}}$. 
Example 3.1. Let $\sigma=865347129 \in S_{9}(132)$. Then $\sigma^{7}$ is defined, and is equal to 876534129 . The permutation $\sigma$ is shown below on the left, and $\sigma^{7}$ is shown below on the right.
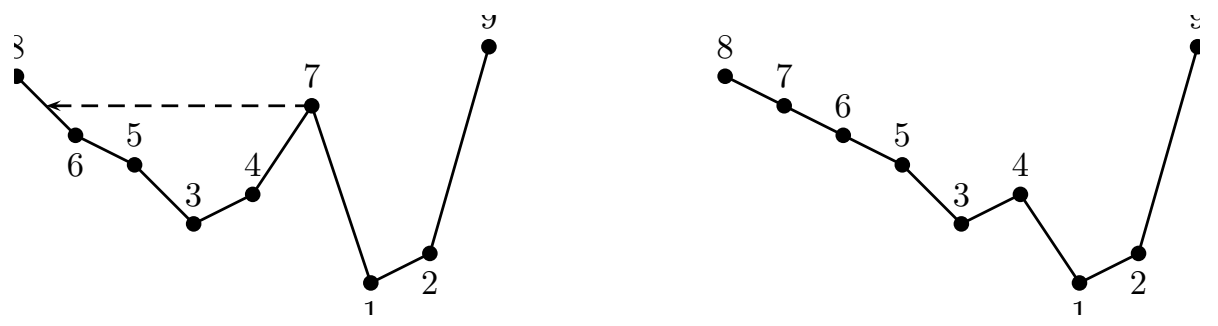

Note that if $\sigma \in S_{n}(132)$ and $\sigma^{\sigma_{j}}$ is defined for some $j$, then $\sigma$ can be broken into five parts (dependent on $j$ ) as follows:

- The set of numbers $\left\{\sigma_{1}, \ldots, \sigma_{i-1}\right\}$, which we denote by $L$ for left. Every number in $L$ is greater than $\sigma_{j}$.

- $\sigma_{i}$.

- The set of numbers $\left\{\sigma_{i+1}, \ldots, \sigma_{j-1}\right\}$, which we denote by $M$ for middle (this set is empty if $j=i+1)$. Every number in $M$ is less than $\sigma_{i}$.

- $\sigma_{j}$.

- The set of numbers $\left\{\sigma_{j+1}, \ldots, \sigma_{n}\right\}$, which we denote by $R$ for right. Each number in this set is either less than all numbers in the set $M \cup \sigma_{i}$, or is greater than $\sigma_{j}$.

Example 3.2. Let $\sigma=865347129 \in S_{9}(132)$ (see Example 3.1), and let $\sigma_{j}=7$. Then $L=\{8\}$, $M=\{5,3,4\}$, and $R=\{1,2,9\}$.

Proposition 3.3. Suppose $\sigma \in S_{n}(132)$ and that for some $j, \phi=\sigma^{\sigma_{j}}$ is defined. Then $\phi \in S_{n}(132)$.

Proof: Suppose for a contradiction that $\phi \notin S_{n}(132)$. Then $\sigma_{j}$ must occur as an element in a 132 pattern in $\phi$. If $\sigma_{j}$ occurs as 1, then the elements that occur as 3 and 2 must be contained in $R$. This is the desired contradiction that $\sigma$ would not be 132-avoiding, since the same triple of integers form a 132 pattern in $\sigma$. If $\sigma_{j}$ occurs as 3 or 2 we also have a contradiction since $\sigma_{1} \ldots \sigma_{i-1}$ are all greater than $\sigma_{j}$.

Proposition 3.4. For any $n \geq 2$ and any $\sigma \in S_{n}(132)-\{n(n-1) \ldots 1\}$, there exists an index $j$ such that $\sigma^{\sigma_{j}}$ is defined.

Proof: Since $\sigma$ is not the permutation $n(n-1) \ldots 1$, there is a minimal $j$ such that $\sigma_{j}>\sigma_{j-1}$. Then permutation $\sigma^{\sigma_{j}}$ is defined since $\sigma_{1} \ldots \sigma_{j-1}$ is decreasing.

Suppose $\tau \in S_{k}(132)$ and $n$ is an integer greater than or equal to $k$. Let $\mathcal{O}_{n}(\tau)$ denote the set of occurrences of $\tau$ in permutations $\sigma \in S_{n}(132)$ such that $n$ is an element in the occurrence, and let $\mathcal{O}_{n}^{\prime}(\tau)$ 
be the set of occurrences of the pattern $\tau$ in permutations $\sigma \in S_{n}(132)$ such that $n$ is not an element in the occurrence. Then

$$
A_{n}(\tau)=\left|\mathcal{O}_{n}(\tau)\right|+\left|\mathcal{O}_{n}^{\prime}(\tau)\right| .
$$

We denote an occurrence of $\tau$ in some $\sigma=\sigma_{1} \ldots \sigma_{n}$ by a pair $\left(\sigma, \sigma_{s_{1}} \ldots \sigma_{s_{k}}\right)$ in which $\left\{\sigma_{s_{1}}, \ldots, \sigma_{s_{k}}\right\}$ are the elements in the occurrence of $\tau$.

Given permutations $\tau$ of length $k$ and $\mu$ of length $l$, we define permutations $\tau \oplus \mu$ and $\tau \ominus \mu$ each of length $k+l$ as follows

$$
\begin{aligned}
& (\tau \oplus \mu)_{i}= \begin{cases}\tau_{i}, & \text { if } 1 \leq i \leq k, \\
\mu_{i}+k, & \text { if } k+1 \leq i \leq k+l,\end{cases} \\
& (\tau \ominus \mu)_{i}= \begin{cases}\tau_{i}+l, & \text { if } 1 \leq i \leq k, \\
\mu_{i}, & \text { if } k+1 \leq i \leq k+l .\end{cases}
\end{aligned}
$$

Theorem 3.5. Suppose that $\mu, \tau \in S_{k}(132)$ and that for some $j, \mu=\tau^{\tau_{j}}$. Then for all $n \geq k$ we have $A_{n}(\mu) \geq A_{n}(\tau)$.

Proof: We proceed by induction. By definition, if $\mu=\tau^{\tau_{j}}$ for some $j$, then $\mu$ and $\tau$ have length greater than or equal to 2. Hence, to prove the base case, we need to prove that if $\mu=\tau^{\tau_{j}}$ for some $j$, where $\mu$ is of length 2 , then $A_{2}(\mu) \geq A_{2}(\tau)$. This is true since $A_{2}(\tau)=A_{2}(\mu)=1$. Now assume by induction that $A_{n^{\prime}}(\mu) \geq A_{n^{\prime}}(\tau)$ holds for every pair $\mu$ and $\tau$ of length $k^{\prime}$ with $\mu=\tau^{\tau_{j}}$ for some $j$, where $k^{\prime} \leq k$ and $n^{\prime}<n$, or $k^{\prime}<k$ and $n^{\prime} \leq n$. Now suppose that $\mu=\tau^{\tau_{j}}$ for some $\mu, \tau$ and $j$, where $\mu \in S_{k}(132)$. We will show that $A_{n}(\mu) \geq A_{n}(\tau)$.

Note that the sets $L, M$ and $R$ that we will mentioned in this proof are subsets of $\left\{\tau_{1}, \ldots, \tau_{k}\right\}$. We will consider occurrences of the pattern $\tau$ contained in permutations $\sigma \in S_{n}(132)$. We will consider the following two cases separately.

a) The set of occurrences of $\tau$ contained in $\mathcal{O}_{n}^{\prime}(\tau)$.

b) The set of occurrences of $\tau$ contained in $\mathcal{O}_{n}(\tau)$. In this case $n$ may occur as $\tau_{j}$ if and only if $L$ is empty, and the elements in $R$ are all less than the elements in $M \cup\left\{\tau_{i}\right\}$.

To show that $A_{n}(\mu) \geq A_{n}(\tau)$ we first show, in part a), by induction that $\left|\mathcal{O}_{n}^{\prime}(\tau)\right| \leq\left|\mathcal{O}_{n}^{\prime}(\mu)\right|$, and we then show, in part b), that $\left|\mathcal{O}_{n}(\tau)\right| \leq\left|\mathcal{O}_{n}(\mu)\right|$.

First we consider case a), i.e., occurrences of $\tau$ in $\mathcal{O}_{n}^{\prime}(\tau)$. Suppose that for some $\xi \leq i$, there exists at least one occurrence of $\tau$ in some permutation $\sigma \in S_{n}(132)$, so that when $\sigma$ is written in one-line notation:

- $n$ is to the left of the element that occurs as $\tau_{\xi}$, and

- if $\xi \neq 1$, then the element that occurs as $\tau_{\xi-1}$ is to the left of $n$. 
Given any such occurrence in which $\sigma_{\alpha+1}=n$, the pattern which is order isomorphic to the word $\tau_{1} \ldots \tau_{\xi-1}$ occurs within the permutation which is order isomorphic to the word $\sigma_{1} \ldots \sigma_{\alpha} \in S_{\alpha}(132)$, and the pattern $\tau_{\xi} \ldots \tau_{k}$ occurs within the permutation $\sigma_{\alpha+2} \ldots \sigma_{n} \in S_{n-\alpha-1}(132)$. Also, given an occurrence of the pattern which is order isomorphic to the word $\tau_{1} \ldots \tau_{\xi-1}$ within a permutation $\theta$ in $S_{\alpha}(132)$, and an occurrence of $\tau_{\xi} \ldots \tau_{k}$ within a permutation $\phi$ in $S_{n-\alpha-1}(132)$, we may concatenate these occurrences to form an occurrence of $\tau$ in the permutation $\sigma=(\theta \oplus 1) \ominus \phi$. Hence, the number of such occurrences of $\tau$ in permutations $\sigma \in \mathcal{O}_{n}^{\prime}(\tau)$ in which $\sigma_{\alpha+1}=n$ is given by

$$
A_{\alpha}\left(\tau_{1} \ldots \tau_{\xi-1}\right) A_{n-\alpha-1}\left(\tau_{\xi} \ldots \tau_{k}\right) .
$$

Summing this over all possible values of $\alpha$ gives the total number of such occurrences (with respect to this value of $\xi$ ) of $\tau \in \mathcal{O}_{n}^{\prime}(\tau)$ :

$$
\sum_{\alpha=\xi-1}^{n+\xi-k-2} A_{\alpha}\left(\tau_{1} \ldots \tau_{\xi-1}\right) A_{n-1-\alpha}\left(\tau_{\xi} \ldots \tau_{k}\right) .
$$

In the case that $\xi=1$, so that $\tau_{1} \ldots \tau_{\xi-1}$ is the empty word, recall that the term $A_{\alpha}\left(\tau_{1} \ldots \tau_{\xi-1}\right)$ equals $\left|S_{\alpha}(132)\right|$, and that $\left|S_{0}(132)\right|=1$. We will now consider, for this value of $\xi$, occurrences of $\mu$ is permutation in $\mathcal{O}_{n}^{\prime}(\mu)$ in which $n$ lies between $\mu_{\xi-1}$ and $\mu_{\xi}$ (or to the left of $\mu$ if $\xi=1$ ). By a similar argument, the number of such occurrences of $\mu$ in all possible $\sigma \in S_{n}(132)$ is the sum

$$
\sum_{\alpha=\xi-1}^{n+\xi-k-2} A_{\alpha}\left(\mu_{1} \ldots \mu_{\xi-1}\right) A_{n-1-\alpha}\left(\mu_{\xi} \ldots \mu_{k}\right) .
$$

Now, $\mu_{1} \ldots \mu_{\xi-1}=\tau_{1} \ldots \tau_{\xi-1}$, so that $A_{\alpha}\left(\mu_{1} \ldots \mu_{\xi-1}\right)=A_{\alpha}\left(\tau_{1} \ldots \tau_{\xi-1}\right)$ for all $\alpha$ in the summation. By induction, we have that

$$
A_{n-1-\alpha}\left(\tau_{\xi} \ldots \tau_{k}\right) \leq A_{n-1-\alpha}\left(\mu_{\xi} \ldots \mu_{k}\right)
$$

for all $\alpha$ in the summation, since $\mu_{\xi} \ldots \mu_{k}=\tau_{\xi} \ldots \tau_{k}^{\tau_{j}}$. Therefore

$$
\begin{gathered}
\sum_{\alpha=\xi-1}^{n+\xi-k-2} A_{\alpha}\left(\tau_{1} \ldots \tau_{\xi-1}\right) A_{n-1-\alpha}\left(\tau_{\xi} \ldots \tau_{k}\right) \\
\leq \sum_{\alpha=\xi-1}^{n+\xi-k-2} A_{\alpha}\left(\mu_{1} \ldots \mu_{\xi-1}\right) A_{n-1-\alpha}\left(\mu_{\xi} \ldots \mu_{k}\right)
\end{gathered}
$$

holds for all $\xi \leq i$ such that $n$ may lie between $\tau_{\xi-1}$ and $\tau_{\xi}$.

Note that it is not possible for $n$ to lie between $\tau_{\xi-1}$ and $\tau_{\xi}$ if $\xi \in\{i+1, \ldots, j\}$. Suppose that for some $\xi \geq j$, there exists at least one occurrence of $\tau$ in some permutation $\sigma \in S_{n}(132)$, so that when $\sigma$ is written in one-line notation:

- $n$ is to the right of the element that occurs as $\tau_{\xi-1}$, and 
- if $\xi-1 \neq k$, then the element that occurs as $\tau_{\xi}$ is to the right of $n$.

For this value of $\xi$, we can again consider occurrences of $\mu$ is $\mathcal{O}_{n}^{\prime}(\mu)$ in which $n$ lies between $\mu_{\xi-1}$ and $\mu_{\xi}$. Note that if $\xi-1=j$, then $\mu_{\xi-1}=\tau_{j-1}$. By a similar argument to that given when $\xi \leq i$, we can deduce that the number of such occurrences of the pattern $\tau$ is less than or equal to the number of such occurrences of the pattern $\mu$. Since we have considered all possible occurrences of $\tau$ in $\mathcal{O}_{n}^{\prime}(\tau)$, we have shown that

$$
\left|\mathcal{O}_{n}^{\prime}(\tau)\right| \leq\left|\mathcal{O}_{n}^{\prime}(\mu)\right|
$$

We now consider case b), i.e., occurrences of $\tau$ in $\mathcal{O}_{n}(\tau)$. Recall that $\mathcal{O}_{n}(\tau)$ is the set of occurrences of $\tau$ in permutations in $S_{n}(132)$ in which $n$ is an element of the occurrence. For this part, we will show that $\left|\mathcal{O}_{n}(\tau)\right| \leq\left|\mathcal{O}_{n}(\mu)\right|$ in two cases. The first case is when $\tau_{j}$ is not equal to $k$, and we prove this case by induction. The second case is when $\tau_{j}=k$, so that for all elements in $\mathcal{O}_{n}(\tau)$ we must have $n$ occur as $\tau_{j}$. In this case we define a map from $\mathcal{O}_{n}(\tau)$ to $\mathcal{O}_{n}(\mu)$ and show that this map in an injection.

Suppose that $\tau_{j} \neq k$, so that $n$ may occur as an element in $L$ or $R$ only. Suppose that for some $\xi \leq i-1$, there exists an occurrence of $\tau$ in which $n$ occurs as $\tau_{\xi}$. Then there exists an occurrence of $\mu$ in $\mathcal{O}_{n}(\mu)$ in which $n$ occurs as $\mu_{\xi}$. The number of such occurrences of the pattern $\tau$ in all possible $\sigma \in S_{n}(132)$ is

$$
\sum_{\alpha=\xi-1}^{n+\xi-k-1} A_{\alpha}\left(\tau_{1} \ldots \tau_{\xi-1}\right) A_{n-1-\alpha}\left(\tau_{\xi+1} \ldots \tau_{k}\right),
$$

and the number of such occurrences of the pattern $\mu$ in all possible $\sigma \in S_{n}(132)$ is

$$
\sum_{\alpha=\xi-1}^{n+\xi-k-1} A_{\alpha}\left(\mu_{1} \ldots \mu_{\xi-1}\right) A_{n-\alpha-1}\left(\mu_{\xi+1} \ldots \mu_{k}\right) .
$$

Since $\tau_{1} \ldots \tau_{\xi-1}=\mu_{1} \ldots \mu_{\xi-1}$ we have that $A_{\alpha}\left(\tau_{1} \ldots \tau_{\xi-1}\right)=A_{\alpha}\left(\mu_{1} \ldots \mu_{\xi-1}\right)$ for all $\alpha$ in the summation. By the inductive hypothesis $A_{n-1-\alpha}\left(\tau_{\xi+1} \ldots \tau_{k}\right) \leq A_{n-1-\alpha}\left(\mu_{\xi+1} \ldots \mu_{k}\right)$ for all $\alpha$ in the summation since $\mu_{\xi+1} \ldots \mu_{k}=\tau_{\xi+1} \ldots \tau_{k}^{\tau_{j}}$. Hence

$$
\begin{gathered}
\sum_{\alpha=\xi-1}^{n+\xi-k-1} A_{\alpha}\left(\tau_{1} \ldots \tau_{\xi-1}\right) A_{n-1-\alpha}\left(\tau_{\xi+1} \ldots \tau_{k}\right) \\
\leq \sum_{\alpha=\xi-1}^{n+\xi-k-1} A_{\alpha}\left(\mu_{1} \ldots \mu_{\xi-1}\right) A_{n-\alpha-1}\left(\mu_{\xi+1} \ldots \mu_{k}\right) .
\end{gathered}
$$

Suppose that for some $\xi \geq j+1$, that there is at least one occurrence of $\tau$ in $\mathcal{O}_{n}(\tau)$ in which $n$ occurs as $\tau_{\xi}$. Then there exists at least one occurrence of $\mu$ in $\mathcal{O}_{n}(\mu)$ in which $n$ occurs as $\mu_{\xi}$. By the same argument as in the case where $\xi \leq i-1$, we have that the number of such occurrences of the pattern $\tau$ is less than or equal to the number of such occurrences of the pattern $\mu$. 
We now consider elements of $\mathcal{O}_{n}(\tau)$ in which $n$ occurs as $\tau_{j}$. Note that this case is possible if and only if $\tau_{i}=\tau_{1}$ (i.e., $L$ is empty), and all elements of $R$ are less than elements in $M \cup \tau_{i}$. For such $\tau$ we will define a map

$$
\psi: \mathcal{O}_{n}(\tau) \rightarrow \mathcal{O}_{n}(\mu)
$$

We will argue that this map in injective, and therefore, the number of such occurrences of the pattern $\tau$ is less than or equal to the number of such occurrences of the pattern $\mu$.

Fix an element $\left(\sigma, \sigma_{s_{1}} \ldots \sigma_{s_{k}}\right) \in \mathcal{O}_{n}(\tau)$ such that $\sigma_{s_{j}}=n$. Consider the earliest index $\alpha \in\left[s_{j}-1\right]$ such that:

- the occurrence of the elements in $M \cup\left\{\tau_{i}\right\}$ in $\sigma$ are in the set $\left\{\sigma_{1}, \ldots, \sigma_{\alpha}\right\}$, and

- each element in $\left\{\sigma_{1}, \ldots, \sigma_{\alpha}\right\}$ is greater than all the elements in the set $\left\{\sigma_{\alpha+1}, \ldots, \sigma_{n}\right\}-\left\{\sigma_{s_{j}}\right\}$.

Note that there must exist such an index $\alpha$ since $s_{j}-1$ satisfies the above two conditions. Note also that since $\sigma \in S_{n}(132)$, the elements $\sigma_{\alpha+1}, \ldots, \sigma_{s_{j}-1}$ (we take this set to be empty if $\sigma_{\alpha}=\sigma_{s_{j}-1}$ ) are an interval in the set of integers $[n]$. Let $u$ be the permutation pattern $\sigma_{1} \ldots \sigma_{\alpha}$, let $v$ be the permutation pattern $\sigma_{\alpha+1} \ldots \sigma_{s_{j}-1}$, and let $w$ be the permutation pattern $\sigma_{s_{j}+1} \ldots \sigma_{n}$. We let

$$
\psi\left(\left(\sigma, \sigma_{s_{1}} \ldots \sigma_{s_{k}}\right)\right)=\left(\bar{\sigma}, \bar{\sigma}_{t_{1}} \ldots \bar{\sigma}_{t_{k}}\right)
$$

where

$$
\bar{\sigma}=((v \oplus 1) \ominus u) \ominus w
$$

with the occurrence $\bar{\sigma}_{t_{1}}, \ldots, \bar{\sigma}_{t_{k}}$ of $\mu$ in $\bar{\sigma}$ is given by $n$, followed by the entries in the patterns $u$ and $w$ in $\bar{\sigma}$ that give the occurrence of the elements

$$
\tau_{1}, \ldots, \tau_{j-1}, \tau_{j+1}, \ldots, \tau_{n}
$$

in $u$ and $w$ in $\sigma$ (see Example 3.6.

Now $\bar{\sigma}$ is clearly in $S_{n}(132)$. To show that $\psi$ in injective, we show that for any occurrence of $\mu$ in the image of this map, we can recover the occurrence of $\tau$ that mapped to it uniquely. Suppose $\psi\left(\left(\sigma, \sigma_{s_{1}} \ldots \sigma_{s_{k}}\right)\right)=\left(\bar{\sigma}, \bar{\sigma}_{t_{1}} \ldots \bar{\sigma}_{t_{k}}\right)$, and consider the earliest index $\beta \in[n]$ such that:

- the occurrence of the elements in $M \cup\left\{\tau_{i}\right\}$ in $\bar{\sigma}$ are in the set $\left\{\bar{\sigma}_{1}, \ldots, \bar{\sigma}_{\beta}\right\}$,

- the occurrence of the elements in $R$ in $\bar{\sigma}$ are in the set $\left\{\bar{\sigma}_{\beta+1}, \bar{\sigma}_{\beta+2}, \ldots, \bar{\sigma}_{n}\right\}$,

- each element in $\left\{\bar{\sigma}_{1}, \ldots, \bar{\sigma}_{\beta}\right\}$ is greater than all the elements in the set $\left\{\bar{\sigma}_{\beta+1}, \ldots, \bar{\sigma}_{n}\right\}$.

Note that there must exist such an index $\beta$ since there was an index $\alpha$ that satisfied these conditions in $\sigma$. Suppose that $\bar{\sigma}_{l}=n$. Then the permutation pattern $\bar{\sigma}_{1}, \ldots, \bar{\sigma}_{l-1}$ is denoted by $v$. We denote the permutation pattern $\bar{\sigma}_{l+1}, \ldots, \bar{\sigma}_{\beta}$ by $u$, and we denote the permutation pattern $\bar{\sigma}_{\beta+1} \ldots \bar{\sigma}_{n}$ by $w$. Then

$$
\bar{\sigma}=((v \oplus 1) \ominus u) \ominus w
$$


and the occurrence of $\tau$ that maps to $\left(\bar{\sigma}, \bar{\sigma}_{t_{1}} \ldots \bar{\sigma}_{t_{k}}\right)$ must be given by

$$
\sigma=((u \ominus v) \oplus 1) \ominus w
$$

where $n$ occurs as $\tau_{j}$, and the entries in $u$ and $w$ that corresponded to the occurrence of $\mu_{2} \ldots \mu_{n}$ in $\bar{\sigma}$ give the occurrence of $\tau_{1} \ldots \tau_{j-1} \tau_{j+1} \ldots \tau_{n}$ in $\sigma$ (see Example 3.6. This is exactly $\left(\sigma, \sigma_{s_{1}} \ldots \sigma_{s_{k}}\right)$.

Example 3.6. We give an example of the map $\psi$ given in the proof of Theorem 3.5. Let $k=6$ and let $n=13$. Let $\tau$ be the permutation 534612, and let $\mu=\tau^{6}=653412$. Let

$$
((11,12,8,7,9,6,10,5,4,13,2,1,3),(12,7,9,13,2,3))
$$

be an occurrence of $\tau$ in $\sigma=(11,12,8,7,9,6,10,5,4,13,2,1,3)$. The occurrence $(\sigma, \tau)$ is shown below in Figure $1(a)$, where we have included a dot above elements in the occurrence of $\tau$. The index $s_{j}$ is equal to 10 , and $\sigma_{10}=13$ occurs as $\tau_{j}=6$. The pattern $u$ is given by $(11,12,8,7,9,6,10)$, the pattern $v$ is given by $(5,4)$, and the pattern $w$ is given by $(2,1,3)$. Then the occurrence of $\mu$ in the permutation $\bar{\sigma}$ is given by

$$
((12,11,13,9,10,6,5,7,4,8,2,1,3),(13,10,5,7,2,3)),
$$

and is shown in Figure 1(b) below.

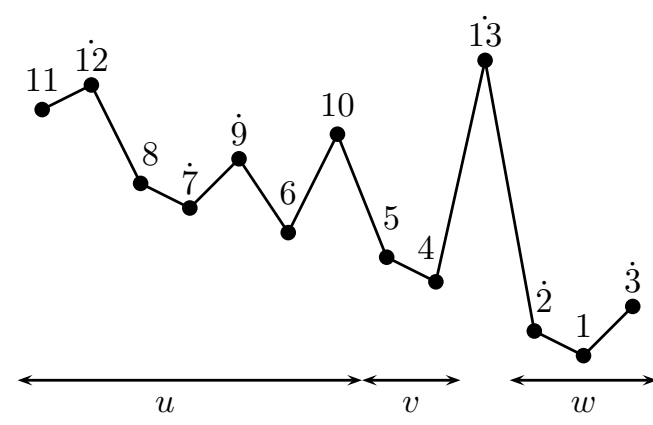

(a)

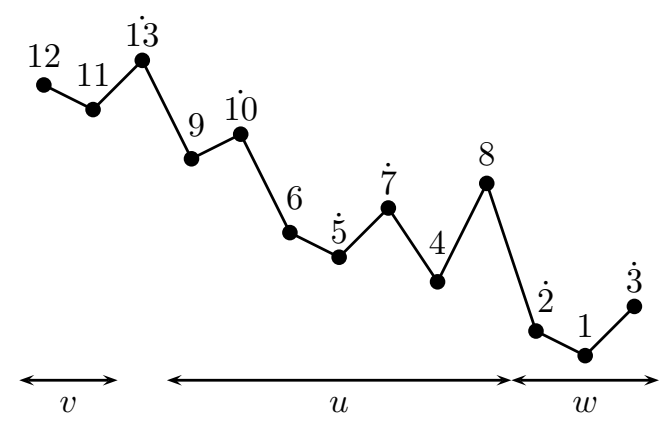

(b)

Fig. 1:

\section{Proof of the main theorem}

In this section, we prove the main theorem, Theorem 4.6. Before proving Theorem 4.6, we describe the binary tree $T(\sigma)$ associated to a 132-avoiding permutation $\sigma$, and we then describe the difference in the structure of $T(\tau)$ and $T(\mu)$ where $\mu=\tau^{\tau_{j}}$ for some $j$.

The set of all 132-avoiding permutations of length $n$ is in bijection with the set of all binary trees with $n$ vertices, denoted $\mathcal{T}_{n}$. This is a well-known result, and it is described in detail in [6]. We will briefly 
describe this bijection. For any tree $T \in \mathcal{T}_{n}$, we label the vertices as follows. We visit each of the vertices of the tree in pre-order (root, left subtree, right subtree), labelling the $i$ th vertex visited by $n+1-i$. Given $T$ with such a labelling, we recover its corresponding permutation $\sigma=\sigma_{1} \ldots \sigma_{n} \in S_{n}(132)$ by visiting the vertices in in-order (left subtree, root, right subtree), letting the $i$ th vertex visited be the value of $\sigma_{i}$. Following the notation of [6], for any $\sigma \in S_{n}(132)$ we denote its associated binary tree by $T(\sigma)$. We will also assume that the trees come with the pre-order labeling described. Rudolph defines the spines of a binary tree $T$ to be connected components of $T$ when all edges connecting left children to their parents are deleted. She also defines the spine structure of $T$ to be the sequence $\left\langle a_{1}, \ldots, a_{s}\right\rangle$, where the $a_{i}$ are the number of vertices in each of the spines in $T$ listed in descending order. We also denote the spine structure of a tree $T$ by $S(T)$, and call the numbers $a_{i}$ the parts of $S(T)$. To make discussions easier, we also define the spine structure of a 132-avoiding permutation $\tau$ to be the spine structure of the binary tree $T(\tau)$. Define the relation $\leq_{R}$ called the refinement order on the set of all spine structures whose parts sum to $n$ as follows. If $\left\langle a_{1}, \ldots, a_{s}\right\rangle$ and $\left\langle b_{1}, \ldots, b_{t}\right\rangle$ are two spine structures such that $\sum_{i=1}^{s} a_{i}=\sum_{i=1}^{t} b_{i}=n$, then $\left\langle a_{1}, \ldots, a_{s}\right\rangle \geq_{R}\left\langle b_{1}, \ldots, b_{t}\right\rangle$ (or equivalently $\left\langle b_{1}, \ldots, b_{t}\right\rangle \leq_{R}\left\langle a_{1}, \ldots, a_{s}\right\rangle$ ) if $\left\langle a_{1}, \ldots, a_{s}\right\rangle$ can be obtained from $\left\langle b_{1}, \ldots, b_{t}\right\rangle$ by merging subsets of its parts. For example $\langle 5,4,3\rangle \geq_{R}\langle 3,3,2,2,1,1\rangle$ since it can be obtained by merging the subset of parts $\{3,2\}$ and the subset of parts $\{2,1,1\}$.

Suppose $G$ is a graph with vertex set $S$. An induced subgraph $H$ of $G$ is a graph with vertex set a subset of $S$, such that two vertices are adjacent in $H$ if and only if they are adjacent in $G$. A subtree $H$ of a binary tree graph $G$ is an induced subgraph that is itself a binary tree graph, in which the root of $H$ is the closest vertex of $H$ to the root of $G$.

The following two propositions describe the child/parent relation between vertices in $T(\sigma)$ for a permutation $\sigma$. They are used to understand the proof of Proposition 4.3, which describes how $T\left(\sigma^{\sigma_{j}}\right)$ is obtained from $T(\sigma)$ when $\sigma^{\sigma_{j}}$ is defined.

Proposition 4.1. Suppose $\sigma=\sigma_{1} \ldots \sigma_{n} \in S_{n}(132)$. For any index $\alpha \in[n]$ such that $\sigma_{\alpha} \in[n-1]$, let $\sigma_{\beta}$ be the least integer such that:

- $\sigma_{\beta}>\sigma_{\alpha}$,

- if $\beta<\alpha$, then $\sigma_{\beta+1}, \ldots, \sigma_{\alpha-1}$ (this set is empty if $\beta=\alpha-1$ ) are all less than $\sigma_{\alpha}$,

- if $\beta>\alpha$, then $\sigma_{\alpha+1}, \ldots, \sigma_{\beta-1}$ (this set is empty if $\beta=\alpha+1$ ) are all less than $\sigma_{\alpha}$.

Then $\sigma_{\beta}$ is the parent vertex of $\sigma_{\alpha}$ in $T(\sigma)$.

Proof: Suppose that $\sigma_{m}=n$. Since $\sigma \in S_{n}(132)$ the words $\sigma_{1} \ldots \sigma_{m-1}$ and $\sigma_{m+1} \ldots \sigma_{n}$ are both 132-avoiding. Now $T(\sigma)$ has root $n$, and the left child of $n$ is the root of the subtree $T\left(\sigma_{1} \ldots \sigma_{m-1}\right)$, and the right child of $n$ is the root of the subtree $T\left(\sigma_{m+1} \ldots \sigma_{n}\right)$. We assume by way of induction, that the proposition is true for all 132-avoiding permutations of length less than $n$. The proposition is clearly true for permutations of length 1 . By the inductive hypothesis, the proposition holds for $T\left(\sigma_{1} \ldots \sigma_{m-1}\right)$ and $T\left(\sigma_{m+1} \ldots \sigma_{n}\right)$. Then, since the root of $T\left(\sigma_{1} \ldots \sigma_{m-1}\right)$ is the largest integer in the set $\left\{\sigma_{1}, \ldots, \sigma_{m-1}\right\}$, and the root of $T\left(\sigma_{m+1} \ldots \sigma_{n}\right)$ is the largest integer in the set $\left\{\sigma_{m+1}, \ldots, \sigma_{n}\right\}$, the condition of the proposition holds for all vertices in $T(\sigma)$, so that the proposition holds for all $n$.

We may also rephrase this result as a condition for being a child, rather than a parent: 
Proposition 4.2. Suppose $\sigma=\sigma_{1} \ldots \sigma_{n} \in S_{n}(132)$. For any index $\alpha \in[n]$ such that $\sigma_{\alpha}>1$, let $\beta$ be an index such that $\sigma_{\beta}<\sigma_{\alpha}$, and either:

- $\beta<\alpha$, and $\sigma_{\beta}$ is the greatest integer such that $\left\{\sigma_{\beta+1}, \ldots, \sigma_{\alpha-1}\right\}$ (this set is empty if $\beta=\alpha-1$ ) are all less than $\sigma_{\beta}$,

- $\beta>\alpha$, and $\sigma_{\beta}$ is the greatest integer such that $\left\{\sigma_{\alpha+1}, \ldots, \sigma_{\beta-1}\right\}$ (this set is empty if $\beta=\alpha+1$ ) are all less than $\sigma_{\beta}$.

Then $\sigma_{\beta}$ is a child vertex of $\sigma_{\alpha}$ in $T(\sigma)$.

Suppose $\sigma$ is a permutation in $S_{n}(132)$ such that $\sigma^{\sigma_{j}}$ is defined for some $j$. Using Propositions 4.1 and 4.2. we can deduce the following about the construction of $T(\sigma)$ :

- The vertex $\sigma_{i}$ is the left child of $\sigma_{j}$.

- The vertex $\sigma_{i}$ has no left child.

- The subtree of $T$ consisting of the right child of $\sigma_{i}$ and its descendants is the tree $T\left(\sigma_{i+1} \ldots \sigma_{j-1}\right)$.

- Suppose there is a largest integer $m$ such that all elements in the set $\left\{\sigma_{j+1}, \ldots, \sigma_{m}\right\}$ are all less than $\sigma_{j}$. Then the right child of $\sigma_{j}$ is the root of the tree $T\left(\sigma_{j+1} \ldots \sigma_{m}\right)$. If $j=n$ or $\sigma_{j+1}>\sigma_{j}$, so that there is no such integer $m$, then $\sigma_{j}$ has no right child (that is, the tree $T\left(\sigma_{j+1} \ldots \sigma_{m}\right)$ is empty).

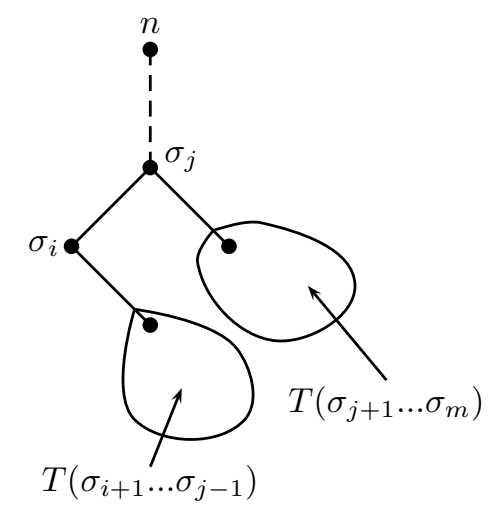

Fig. 2: The tree $T(\sigma)$ for some $\sigma \in S_{n}(132)$ such that $\sigma^{\sigma_{j}}$ is defined for some $j$. The vertex $\sigma_{j}$ may be the left or right child of its parent vertex.

The following proposition describes how we may alter $T(\sigma)$ to obtain $T(\phi)$ where $\phi=\sigma^{\sigma_{j}}$ for some $j$. Figures 34 and 5 give an example of this process.

Proposition 4.3. Suppose that $\phi=\sigma^{\sigma_{j}}$ is defined for some $j$. Suppose that $m$ is the largest integer such that $\sigma_{j+1}, \ldots, \sigma_{m}$ are all less than $\sigma_{j}$. Then $T(\phi)$ is obtained from $T(\sigma)$ by the following steps: 
(1) Remove the subtrees $T\left(\sigma_{i+1} \ldots \sigma_{j-1}\right)$ and $T\left(\sigma_{j+1} \ldots \sigma_{m}\right)$ from $T(\sigma)$.

(2) Remove the vertex $\sigma_{i}$ from the remaining tree and reattach it as the right child of $\sigma_{j}$.

(3) Reattach the tree $T\left(\sigma_{i+1} \ldots \sigma_{j-1}\right)$ so that its root is the right child of $\sigma_{i}$.

(4) Reattach the tree $T\left(\sigma_{j+1} \ldots \sigma_{m}\right)$ so that its root is the right child of the vertex $\sigma_{j-1}$ (note that $\sigma_{j-1}$ is the smallest integer in the spine of the tree $T\left(\sigma_{i+1} \ldots \sigma_{j-1}\right)$ that contains its root).

Proof: The reader should refer to Propositions 4.1 and 4.2 throughout every step of this proof. Consider all numbers in the set

$$
\left\{\sigma_{1}, \sigma_{2}, \ldots, \sigma_{n}\right\}-\left(\left\{\sigma_{i}, \sigma_{i+1}, \ldots, \sigma_{j-1}\right\} \cup\left\{\sigma_{j+1}, \ldots, \sigma_{m}\right\}\right) .
$$

A vertex is a left (respectively right) child of another vertex $w$ in this set in $T(\sigma)$, if and only if it is a left (respectively right) child of $w$ in $T(\phi)$. This confirms that step (1) is correct, i.e., that the subtree on the vertices

$$
\left\{\sigma_{1}, \sigma_{2}, \ldots, \sigma_{n}\right\}-\left(\left\{\sigma_{i}, \sigma_{i+1}, \ldots, \sigma_{j-1}\right\} \cup\left\{\sigma_{j+1}, \ldots, \sigma_{m}\right\}\right)
$$

is identical in $T(\sigma)$ and $T(\phi)$.

It is clear that $\sigma_{i}$ is the right child of $\sigma_{j}$ in $T(\phi)$. This confirms that step (2) is correct.

A vertex $v$ in the set $\left\{\sigma_{i}, \ldots, \sigma_{j-1}\right\}$ is the left (respectively right) child of another vertex $w$ in this set in $T(\sigma)$, if and only if it is the left (respectively right) child of $w$ in $T(\phi)$. This confirms that step (3) is correct.

A vertex $v$ in the set $\left\{\sigma_{j+1} \ldots \sigma_{m}\right\}$ is a left (respectively right) child of another vertex $w$ in this set in $T(\sigma)$, if and only if it is a left (respectively right) vertex of $w$ in $T(\phi)$. Also, the root of $T\left(\sigma_{j+1} \ldots \sigma_{m}\right)$ is the right child of $\sigma_{j-1}$ in $T(\phi)$. This confirms step 4.

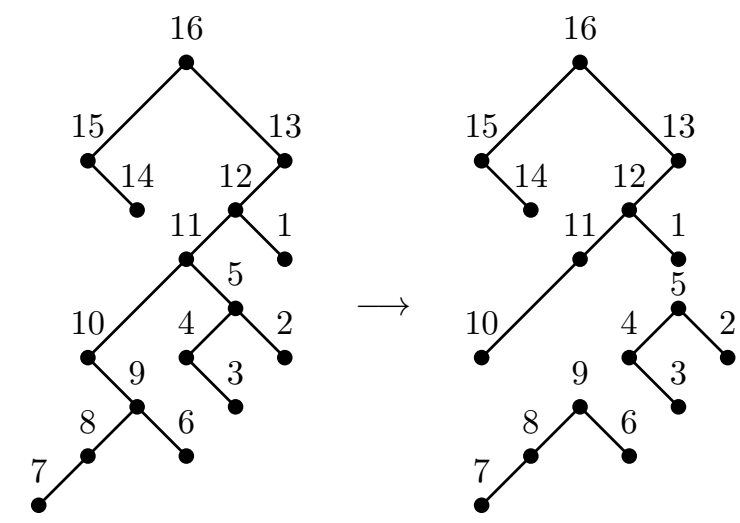

Fig. 3: An example of $T(\sigma)$ for $\sigma=(15,14,16,10,7,8,9,6,11,4,3,5,2,12,1,13)$ in which $\sigma_{i}=10$ and $\sigma_{j}=$ 11 . We remove the subtrees $T\left(\sigma_{i+1} \ldots \sigma_{j-1}\right)=T(7896)$ and $T\left(\sigma_{j+1} \ldots \sigma_{m}\right)=T(4352)$. 


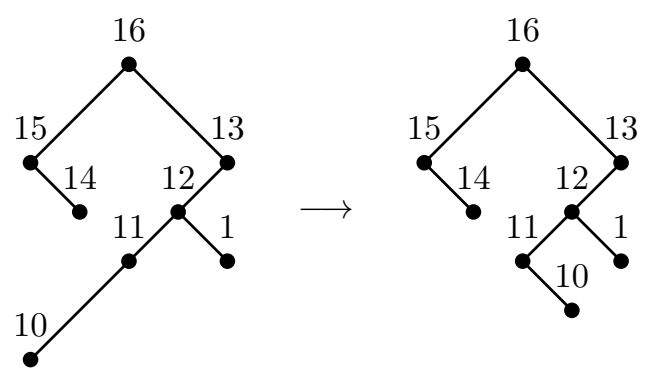

Fig. 4: Reattaching the vertex $\sigma_{i}=10$ so that it is the right child of $\sigma_{j}=11$.
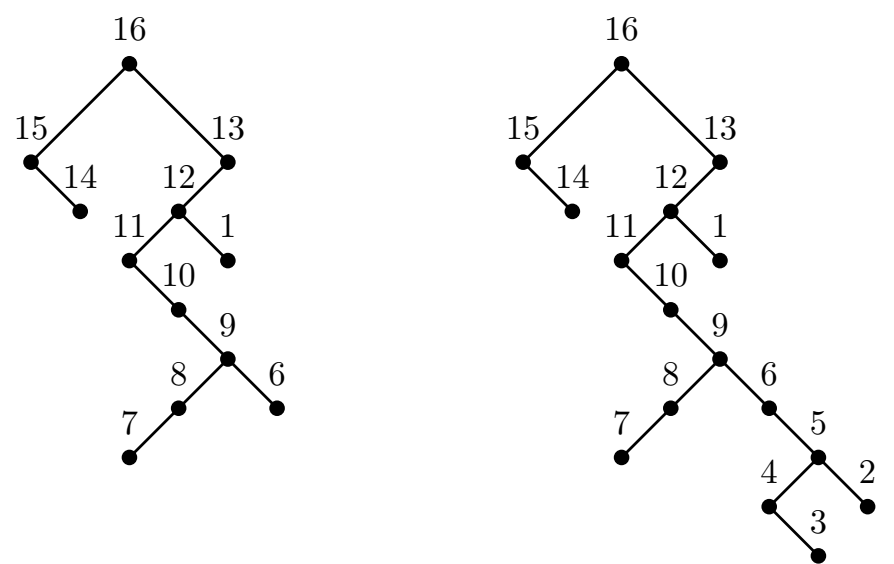

Fig. 5: Left: reattaching the tree $T\left(\sigma_{i+1} \ldots \sigma_{j-1}\right)$. Right: reattaching the tree $T\left(\sigma_{j+1} \ldots \sigma_{m}\right)$.

Proposition 4.4. Suppose that for some $j, \phi=\sigma^{\sigma_{j}}$, and suppose that $S(T(\sigma))=\left\langle a_{1}, \ldots, a_{s}\right\rangle$. Then $S(T(\phi))$ is obtained from the spine structure $\left\langle a_{1}, \ldots, a_{s}\right\rangle$ by merging two parts. That is, there exist indices $l, m$, such that the parts in $S(T(\phi))$ are $\left\{a_{1}, \ldots, a_{s}\right\}-\left\{a_{l}, a_{m}\right\} \cup\left\{a_{l}+a_{m}\right\}$.

Proof: By Proposition 4.3 the spine structure of $T(\phi)$ is obtained from the spine structure of $T(\sigma)$ by merging the spine of $T(\sigma)$ that contains $\sigma_{i}$ and the spine of $T(\sigma)$ that contains $\sigma_{j}$.

The following definition appears in [6]. A left-justified binary tree is is a binary tree in which every vertex that is a right child of its parent does not have a left child.

Proposition 4.5. Suppose that $T_{1}, T_{2} \in \mathcal{T}_{n}$ and the spine structure of $T_{2}$ is obtained from the spine structure of $T_{1}$ by merging two parts. Then there exists $\sigma \in S_{n}(132)$ such that $\phi=\sigma^{\sigma_{j}}$ is defined for some $j$, and such that $T(\sigma)$ has the same spine structure as $T_{1}$, and $T(\phi)$ has the same spine structure as $T_{2}$.

Proof: Suppose that $\left\langle a_{1}, \ldots, a_{s}\right\rangle$ is the spine structure of $T_{1}$, and that $a_{l}$ and $a_{m}$ are merged to give the 
spine structure of $T_{2}$. We will construct a new tree $T \in \mathcal{T}_{n}$ that is left-justified, with the same spine structure as $T_{1}$. If we label the vertices of any left-justified tree with $s$ parts in pre-order, then the vertices $n-s+1, n-s+2, \ldots, n-1, n$ are a path of vertices in this tree, in which each vertex in this sequence is the left child of the following vertex. Let $T$ be such a tree, so that the spine containing vertex $n-s+1$ has $a_{l}$ vertices, and the spine containing vertex $n-s+2$ has $a_{m}$ vertices. The order of the remaining spines in $T$ is irrelevant. Then $T$ is equal to $T(\sigma)$ for some $\sigma \in S_{n}(132)$ such that $\phi=\sigma^{n-s+2}$ is defined, and the tree $T(\phi)$ has the same spine structure as $T_{2}$.

Theorem 4.6. Suppose $\tau$ and $\mu$ are elements of $S_{k}(132)$. If the spine structure of $T(\tau)$ is less than or equal to the spine structure of $T(\mu)$ in refinement order, then for all $n, A_{n}(\tau) \leq A_{n}(\mu)$.

Proof: We may assume that the spine structure of $T(\mu)$ is obtained from the spine structure of $T(\tau)$ by merging two parts, since if this implies $A_{n}(\tau) \leq A_{n}(\mu)$, then the theorem also holds if $S(T(\mu))$ is obtained from $S(T(\tau))$ by merging any subsets of parts. By Proposition 4.5 there exists $\bar{\tau}$ and $j$ such that $\bar{\tau}^{\bar{\tau}_{j}}$ is defined, and such that $S(T(\tau))=S(T(\bar{\tau}))$ and $S(T(\mu))=S\left(T\left(\overline{\bar{\tau}}^{\bar{\tau}_{j}}\right)\right)$. By Theorem $1.1 . A_{n}(\tau)=$ $A_{n}(\bar{\tau})$ and $A_{n}(\mu)=A_{n}\left(\bar{\tau}^{\bar{\tau}_{j}}\right)$. By Theorem $3.5, A_{n}(\bar{\tau}) \leq A_{n}\left(\bar{\tau}^{\bar{\tau}_{j}}\right)$, so that $A_{n}(\tau) \leq A_{n}(\mu)$.

We will now strengthen the result of Theorem 4.6 by showing, in Theorem 4.8, that if $\mu=\tau^{\tau_{j}}$ for $\mu \in S_{k}(132)$, and $n>k$, then $A_{n}(\mu)>A_{n}(\tau)$. The following proposition on spine structures is used to prove Theorem 4.8

Proposition 4.7. Suppose that $\tau \in S_{k}(132)$ and that for some $j, \mu=\tau^{\tau_{j}}$ is defined. Then there exists a permutation $\bar{\tau} \in S_{k}(132)$ such that $\bar{\mu}=\bar{\tau}^{k}$ is defined, $\left.S(T(\tau))=S(T(\bar{\tau}))\right)$ and $S(T(\mu))=S(T(\bar{\mu}))$.

Proof: Suppose the spine of $T(\tau)$ that contains $\tau_{j}$ contains $l$ vertices, and that the spine of $T(\tau)$ that contains $\tau_{i}=\tau_{j}-1$ contains $m$ vertices. Create a binary tree $T$ with the same spine structure as $T(\tau)$ as follows. First, form a left-justified binary tree $T_{1}$ with two spines, one of length $m$ and the other of length $l$, so that the root of the length $l$ spine is the root of $T_{1}$. Form a left-justified binary tree $T_{2}$ whose spines consist of the spines of $T$ that do not contain $\tau_{i}$ or $\tau_{j}$. Form $T$ by adjoining $T_{1}$ and $T_{2}$ so that the root of $T_{2}$ is the left child of the leaf contained in the same spine as $\tau_{i}$ of $T_{1}$. See Figure 6 for an example.

Theorem 4.8. Suppose $\tau \in S_{k}(132)$ and that for some $j, \mu=\tau^{\tau_{j}}$ is defined. Then for any integer $n>k$ we have $A_{n}(\mu)>A_{n}(\tau)$.

Proof: By Proposition 4.7, we may assume that $\tau_{j}=k$. Then it is possible that $n$ occurs as $\tau_{j}$. In order to understand this proof we need to recall the proof of Theorem 3.5. We will show that the injective map from $\mathcal{O}_{n}(\tau)$ to $\mathcal{O}_{n}(\mu)$, defined in the proof of Theorem 3.5, is not onto. This is proves the theorem.

Suppose $n>k$. Then there exists some $\sigma$ in $S_{n}(132)$ which contains the pattern $\mu$, such that $n$ occurs as $\mu_{1}=k, \sigma_{n}=n-1$, and $\sigma_{n}$ is not an element in the occurrence of $\mu$. We require that $\sigma_{1}=n$ in this case. If such an occurrence of $\mu$ is mapped to by an occurrence of $\tau$, then the occurrence of $\tau$ is obtained by shifting $n$ so that entries occurring as elements in $M \cup\left\{\tau_{i}\right\}$ (note $\tau_{i}=\mu_{2}$ ) are to its left, and entries occurring as elements in $R$ are to its right. However such a permutation would not be in $S_{n}(132)$ because $\sigma_{s} n(n-1)$ forms a 132 pattern, where $\sigma_{s}$ occurs as $\mu_{2}$. Hence, in this case, $A_{n}(\mu)>A_{n}(\tau)$. 

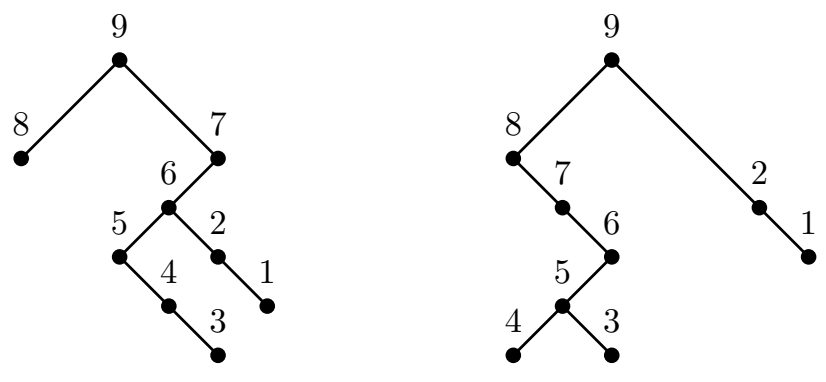

Fig. 6: Let $\tau=895436217 \in S_{9}(132)$, and let $\mu=\tau^{6}$. Then $T(874536921)$ has the same spine structure as $T(\tau)$, and $T\left(874536921^{9}\right)$ has the same spine structure as $T(\mu)$. The tree shown on the left is $T(\tau)$ and the tree shown on the right is $T(874536921)$

\section{A counterexample to Conjecture 1.2}

In this section, we provide a counterexample to Conjecture 1.2 , by showing that for all $n \geq 4$ we have $A_{n}(3241) \leq A_{n}(3421)$. This provides a contradiction since $S(T(3241))=\langle 2,2\rangle$ and $S(T(3421))=$ $\langle 3,1\rangle$ are incomparable in refinement order.

Proposition 5.1. For all $n \geq 4$ we have $A_{n}(3241) \leq A_{n}(3421)$. Furthermore, we have equality if and only if $n=4$.

Proof: We assume by induction that for all $k \in\{4,5, \ldots, n-1\}$ we have $A_{k}(3241) \leq A_{k}(3421)$. This is true for $k=4$ since $A_{4}(3241)=A_{4}(3421)=1$. We will now consider possible occurrences of 3241 and 3421 in permutations in $S_{n}(132)$ for some $n \geq 4$.

For all $n \geq 4$ the following expression holds:

$$
\begin{aligned}
A_{n}(3241) & =2 \sum_{i=4}^{n-1} A_{i}(3241)\left|S_{n-1-i}(132)\right|+\sum_{i=3}^{n-2} A_{i}(324) A_{n-1-i}(1) \\
& +\sum_{i=2}^{n-2} A_{i}(32) A_{n-1-i}(1) .
\end{aligned}
$$

The sum of the first two terms is the number of occurrences of 3241 in which $n$ is not an element in the occurrence. The first term is the number of occurrences in which the element $n$ lies entirely to the right or entirely to the left of elements in the occurrence of 3241, and the second term is the sum of occurrences in which the element $n$ lies between the entry that occurs as 4 and the entry that occurs as 1 . The final term is the number of occurrences in which $n$ occurs as 4 . Also, for all $n \geq 4$ : 


$$
\begin{aligned}
A_{n}(3421) & =2 \sum_{i=4}^{n-1} A_{i}(3421)\left|S_{n-1-i}(132)\right|+\sum_{i=3}^{n-2} A_{i}(342) A_{n-1-i}(1) \\
& +\sum_{i=2}^{n-3} A_{i}(34) A_{n-1-i}(21)+\sum_{i=1}^{n-3} A_{i}(3) A_{n-1-i}(21) .
\end{aligned}
$$

The sum of the first three terms in the summation is the number of occurrences of 3421 in which $n$ is not an element in the occurrence. The first term is the number of occurrences in which the entry $n$ is entirely to the right or entirely to the left of all elements in the occurrence of 3421 . The second term is the number of occurrences of 3421 in which the entry $n$ lies to the right of the entry that occurs as 2 and to the left of the entry that occurs as 1 . The third term is the number of occurrences in which the entry $n$ lies between the entry that occurs as 4 and the entry that occurs as 2 . The fourth term is the number of occurrences in which $n$ occurs as 4 .

By induction, we have that

$$
\sum_{i=4}^{n-1} A_{i}(3241)\left|S_{n-1-i}(132)\right| \leq \sum_{i=4}^{n-1} A_{i}(3421)\left|S_{n-1-i}(132)\right| .
$$

By Bona's result in [2], we have that

$$
\sum_{i=3}^{n-2} A_{i}(324) A_{n-1-i}(1)=\sum_{i=3}^{n-2} A_{i}(342) A_{n-1-i}(1) .
$$

Also,

$$
\sum_{i=2}^{n-2} A_{i}(32) A_{n-1-i}(1)=\sum_{i=1}^{n-3} A_{i}(3) A_{n-1-i}(21) .
$$

Since $\sum_{i=2}^{n-3} A_{i}(34) A_{n-1-i}(21)>0$ this implies that $A_{n}(3241)<A_{n}(3421)$.

Corollary 5.2. There exist permutations $\tau$ and $\mu$ of the same length $k$, such that for all $n \geq k$ we have $A_{n}(\tau) \leq A_{n}(\mu)$, and the spine structure of $T(\tau)$ is incomparable to the spine structure of $T(\mu)$ in refinement order.

The counterexample given in Proposition 5.1 leads naturally to Question 5.3, on the popularity of 132 -avoiding permutations. For all $n$, define the partial order $\leq_{L}$, called the lexicographic order, on the set of partitions of $n$, as follows. For any partitions $\left\langle a_{1}, \ldots, a_{s}\right\rangle$ and $\left\langle b_{1}, \ldots, b_{t}\right\rangle$, where the parts are listed in descending order, we have $\left\langle a_{1}, \ldots, a_{s}\right\rangle \geq_{L}\left\langle b_{1}, \ldots, b_{t}\right\rangle$ if there exists $j \in[s]$ such that $a_{1}=b_{1}, a_{2}=b_{2}, \ldots, a_{j-1}=b_{j-1}$ and $a_{j}>b_{j}$. Note that if $\left\langle a_{1}, \ldots, a_{s}\right\rangle \geq_{R}\left\langle b_{1}, \ldots, b_{t}\right\rangle$ then it follows that $\left\langle a_{1}, \ldots, a_{s}\right\rangle \geq_{L}\left\langle b_{1}, \ldots, b_{t}\right\rangle$.

Question 5.3. Let $\tau$ and $\mu$ be permutations in $S_{k}(132)$. Is it true that if $S(T(\tau)) \leq_{L} S(T(\mu))$, then for all $n \geq k$ we have $A_{n}(\tau) \leq A_{n}(\mu)$ ? If this is true, do we have equality only when $n=k$ ?

Proposition 5.1 demonstrates that the first statement in Question 5.3 is true in the case that $k=4$. 


\section{References}

[1] Miklós Bóna, The Absence of a Pattern and the Occurrences of Another, Discrete Mathematics and Theoretical Computer Science, Vol. 14, 2010, no. 2.

[2] Miklós Bóna, Surprising symmetries in Objects Counted by Catalan Numbers, Electronic Journal of Combinatorics, Vol. 19, 2012, no. 1, p. 62.

[3] Lynn Chua, Krishanu Roy Sankar, Equipopularity classes of 132-avoiding permutations, The Electronic Journal of Combinatorics, Vol. 21, 2014, no. 1.

[4] Joshua Cooper, Combinatorial problems I like, http://www.math.sc.edu/ cooper/ combprob.html, accessed 2013.

[5] Cheyne Homberger, Expected Patterns in Permutation Classes, Electronic Journal of Combinatorics, Vol. 19, no.3.

[6] Kate Rudolph, Pattern Popularity in 132-avoiding Permutations, The Electronic Journal of Combinatorics, Vol. 20, 2013, no. 1, p. 8. 
